\title{
Synovial macrophages shield the joints
}

$\mathrm{CX}_{3} \mathrm{CR} 1^{+}$
synovial
macrophages
formed a
distinct
population
that expressed
several
immune-

related genes
Infiltrating macrophages are important mediators of inflammation in rheumatoid arthritis (RA), but surprisingly little is known about tissue-resident synovial macrophages and whether they have protective or destructive functions during disease. A new study has revealed insights into the previously mysterious lives of synovial macrophages in health and disease, including the identification of a subset of cells that create a protective shield around the joint.

"To visualize synovial macrophages and study their origin and spatiotemporal distribution during steady state and arthritis, we used various reporter mouse strains and fate-mapping approaches together with light sheet fluorescence microscopy," explains corresponding author Gerhard Krönke. "This approach provided us with information on the $3 \mathrm{D}$ distribution of distinct macrophage subsets within the joint."

Two main populations of synovial macrophages emerged from these studies on the basis of expression of the chemokine receptor $\mathrm{CX}_{3} \mathrm{CR} 1$; $\mathrm{CX}_{3} \mathrm{CR}^{+}$lining macrophages and $\mathrm{CX}_{3} \mathrm{CR}^{-}$interstitial macrophages. These macrophages were not derived from circulating monocytes. Instead, $\mathrm{CX}_{3} \mathrm{CR} 1^{-}$interstitial

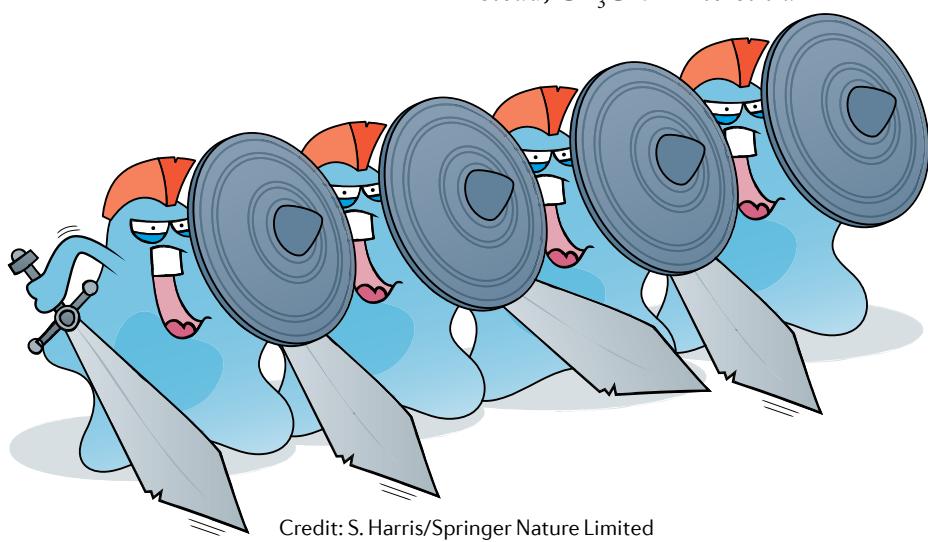

macrophages seemed to be a self-renewing precursor to $\mathrm{CX}_{3} \mathrm{CR}^{+}$ synovial macrophages.

In addition to following the fate of synovial macrophages using various imaging modalities, Krönke and colleagues also use single-cell RNA sequencing to examine the transcriptomes of these macrophage populations. $\mathrm{CX}_{3} \mathrm{CR}^{+}$synovial macrophages formed a distinct population that expressed several immune-related genes. By contrast, $\mathrm{CX}_{3} \mathrm{CR} 1^{-}$ interstitial macrophages could be further divided into several subsets, including actively proliferating cells and a terminally differentiated population characterized by the expression of the putative vascular remodelling hormone RELMa.

"The single and bulk RNA sequencing of the synovium is helpful to recognize the heterogeneity of the synovial macrophage population," states Harris Perlman, an expert on macrophages in arthritis who was not involved in this study. However, as with all genomics studies, the reproducibility of the raw and processed data will need to be verified with other studies."

By comparing single-cell RNA sequencing data on synovial cells from $\mathrm{K} / \mathrm{B} \times \mathrm{N}$ mice with serum transfer-induced arthritis with similar data from the Accelerating Medicines Partnership on synovial cells from patients with RA, the researchers could tentatively match cell populations in mice and in humans. Two of the macrophage populations identified in humans matched tissue-resident synovial macrophages in mice, and two matched infiltrating monocyte-derived macrophages, although an exact match for
$\mathrm{CX}_{3} \mathrm{CR}^{+}$lining macrophages could not be identified in the human data.

Imaging and fate-mapping studies during the development of either serum transfer-induced arthritis or collagen-induced arthritis in mice revealed further insights into the early stages of disease, including a change in the shape of $\mathrm{CX}_{3} \mathrm{CR} 1^{+}$ lining macrophages in response to immune complexes that seems to precede tissue infiltration with immune cells.

"We observed that synovial lining macrophages form a distinct subset that organizes into membrane-like structures around the joint," says Krönke. "Interestingly, this specific macrophage subset shares many features with epithelial cells (including the expression of tight junction proteins) and provides an anti-inflammatory barrier around the joint that hinders immune cell trafficking in a steady state, but 'cracks' open during arthritis."

The researchers plan to further investigate synovial macrophage subsets in different forms of arthritis and to explore the possibility of targeting specific subsets therapeutically.

"The key to future studies will be to understand how each population of synovial macrophages contributes to pathology, and which ones might be the most important for targeted therapy; for example, does one population of synovial macrophages respond to biologics compared with another?" says Perlman.

Joanna Collison ORIGINAL ARTICLE Culemann, S. et al. Locally renewing resident synovial macrophages provide a protective barrier for the joint. Nature https://doi.org/10.1038/s41586-019-1471-1 (2019) RELATED ARTICLE Buckley, C. D. et al. Macrophages form a protective cellular barrier in joints. Nature https://doi.org/10.1038/d41586019-02340-x (2019) 